\title{
BACTERIAL DIVERSITY IN SOME SELECTED AGRICULTURAL FOOD PRODUCTS
}

\author{
Amna Ali*, Muhammad Saleem Haider, Sana Hanif and Muhammad Ashfaq \\ Institute of Agricultural Sciences, University of the Punjab, Quaid-e-Azam Campus, \\ Lahore, 54590, Pakistan
}

Key words: Bacterial diversity, Selected, Agricultural products

\begin{abstract}
A total of 27 bacterial strains were isolated from apple, potato, turnip, onion, cucumber, zinger, lemon, spinach, radish and mint marketted in Labore, Pakistan. Highest frequency of occurrence (4\%) was recorded for each of Enterobacter aggglomerans, Ensifer adhaerens and Bordetella pertussis, Brassica rapa and Kurthia gibsonii showed 30\% frequency of occurrence. Frequency of all other bacterial strain ranged from 10 - 20\%. Highest number (7) of bacterial speices were recorded from lemon and potato while the minimum number (4) was represented by each of apple, onion, ginger, spinach and radish.
\end{abstract}

Bacteria enter plant tissue primarily through the root zone; however, aerial portions of plants, such as flowers, stems, and cotyledons, may also be used for entry. Specifically, the bacteria enter tissues via germinating radicles, secondary root, stomata, or as a result of foliar damage. Bacteria inside a plant may either become localized at the point of entry or spread throughout the plant. These microorganisms can reside within cells, in the intercellular spaces, or in the vascular system (Zinniel et al. 2002). The aim of the present study was to isolate and identify the bacterial species found in different agronomic crops for their diversity.

Ten infected agricultural products viz: apple (Malus domestica L. Borkh.), potato (Solanum tuberosum L.), turnip (Brassica rapa L.), onion (Allium cepa L.), cucumber (Cucumis sativus L., ginger (Zingiber officinale L.), lemon (Citrus limon L. Burm. f.), spinach (Spinacia oleracea L.), radish (Raphanus sativus L.) and mint (Mentha sativa L.) were collected from markets of Lahore, Pakistan, during September to December 2012. The collected materials were stored in sterilized polythylene bags for further investigation. Surface sterilization of samples was done by stepwise washing in $70 \%$ ethanol for $5 \mathrm{~min}$, sodium hypochlorite solution for $5 \mathrm{~min}$, and $70 \%$ ethanol for $30 \mathrm{sec}$, followed by three rinses in sterile distilled water (Ishaq and Khan 2011). Different types of media viz: Luria Bertani agar (LBA), nutrient agar (NA), trypton agar (TA) and yeast extract agar (YEA) were used for isolation and identification (Ali and Naseem 2011, 2012). The surface of infected samples was removed with a sterilized razor blade, and the inner infected tissue was cut into pieces 4 to $6 \mathrm{~mm}$ long and were placed on media plates. Incubation was carried out at $37^{\circ} \mathrm{C}$ for 24 hrs to allow growth of endophytic bacteria. Moreover, fragments of diseased samples were homogenized in $5 \mathrm{ml}$ of sterile saline solution with a blender. The serial dilutions ( $1 \mathrm{ml}$ of $10^{5}$ ) were spread with sterilized spreader onto media (Ishaq and Khan 2011) incubated at $37^{\circ} \mathrm{C}$ for 24 hrs. After incubation, distinct individual colonies were selected and sub-cultured by streaking on an agar plate for purification and preservation. Identification of bacterial species was done by following morphological, microscopic characteristics and biochemical tests and consulting the pertinent literature (Holt et al. 2000, Koneman et al. 1997, Benson 1996). After the identification of bacterial species, following values were determined: (i) percentage of each bacterial isolate in

*Author for correspondence: <write2amna@gmail.com>. 
the sample, (ii) number of occurrences of each bacterial taxa = number of samples colonized by a specific bacteria, (iii) frequency of occurrence of all taxa $(\%)=$ number of samples colonized by a specific bacteria divided by the number of sample examined $\times 100$ (Bajwa et al. 2009).

Table 1. Frequency of occurrence (\%) of bacterial species isolated from agricultural food products.

\begin{tabular}{|c|c|c|c|}
\hline Bacteria & $\begin{array}{l}\text { Food } \\
\text { product }\end{array}$ & $\begin{array}{l}\text { Number of } \\
\text { occurrence }\end{array}$ & $\begin{array}{l}\text { Occurrence } \\
\text { frequency (\%) }\end{array}$ \\
\hline Klebsiella sp. & Apple & 1 & 10 \\
\hline Burkholderia pseudomallei & Apple, lemon, mint & 3 & 30 \\
\hline Yersinia ruckeri & Apple & 1 & 10 \\
\hline Corynebacterium minutissium & Apple, radish & 2 & 20 \\
\hline Bacillus farraginis & Potato, onion, spinach & 3 & 30 \\
\hline Kurthia gibsonii & Potato, turnip, lemon & 3 & 30 \\
\hline Enterobacter aggglomerans & Potato, cucumber, spinach & 4 & 40 \\
\hline Ralstonia solanacearum & Potato & 1 & 10 \\
\hline Azospirillium lipoferum & $"$ & 1 & 10 \\
\hline Acinetobacter lwoffii & Potato, onion & 2 & 20 \\
\hline Peptococcus sp. & Turnip, mint & 2 & 20 \\
\hline Acidovorax facilis & Turnip & 1 & 10 \\
\hline Ensifer adhaerens & Turnip, cucumber, lemon, mint & 4 & 40 \\
\hline Acinetobacter calcoaceticus & Turnip, lemon & 2 & 20 \\
\hline Curtobacterium albidum & Onion, ginger & 2 & 20 \\
\hline Acetobacter aceti & Cucumber & 1 & 10 \\
\hline Acidovorax temperans & $"$ & 1 & 10 \\
\hline Bordetella pertussis & Cucumber, lemon, radish, mint & 4 & 40 \\
\hline Lactococcus lactis & Ginger, radish & 2 & 20 \\
\hline Pantoea sp. & Ginger & 1 & 10 \\
\hline Spirillospora albida & $"$ & 1 & 10 \\
\hline Aerococcus sp. & Lemon & 1 & 10 \\
\hline Syntrophospora sp. & $"$ & 1 & 10 \\
\hline Micrococcus luteus & Spinach & 1 & 10 \\
\hline Proteus vulgaris & $"$ & 1 & 10 \\
\hline Microbacterium lacticum & Radish & 1 & 10 \\
\hline Arthrobacter sp. & Mint & 1 & 10 \\
\hline Total & & 48 & 480 \\
\hline
\end{tabular}

A total of 27 bacterial strains representing 24 genera were isolated from ten different types of agricultural products. Two species belonged to genus Acinetobacter and Acidovorax whereas the rest of the genera Klebsiella sp., Burkholderia pseudomallei, Yersinia ruckeri, Corynebacterium minutissium, Bacillus farraginis, Kurthia gibsonii, Enterobacter aggglomerans, Ralstonia solanacearum, Azospirillium lipoferum, Peptococcus sp., Ensifer adhaerens, Curtobacterium albidum, Acetobacter aceti, Bordetella pertussis, Lactococcus lactis, Pantoea sp., Spirillospora albida, Aerococcus sp., Syntrophospora sp., Micrococcus luteus, Proteus vulgaris, Microbacterium lacticum and Arthrobacter sp. were represented by a single species (Table 1). The 
Table 2. Percentage of composition of bacteria in individual agricultural food products.

\begin{tabular}{|c|c|c|c|c|c|}
\hline $\begin{array}{l}\text { Sample } \\
\text { No. } \\
\end{array}$ & $\begin{array}{l}\text { Substrate/ } \\
\text { host }\end{array}$ & $\begin{array}{l}\text { Place of } \\
\text { collection }\end{array}$ & Bacteria & $\begin{array}{c}\text { No. of } \\
\text { colonies }\end{array}$ & $\begin{array}{c}\% \text { of } \\
\text { bacteria }\end{array}$ \\
\hline \multirow[t]{4}{*}{01} & \multirow[t]{4}{*}{ Apple } & Fruit market, & Klebsiella sp. & 04 & 26 \\
\hline & & \multirow[t]{3}{*}{ Lahore, Cantt. } & Burkholderia pseudomallei & 03 & 20 \\
\hline & & & Yersinia ruckeri & 05 & 33 \\
\hline & & & Corynebacterium minutissium & 03 & 20 \\
\hline \multirow[t]{6}{*}{02} & \multirow[t]{6}{*}{ Potato } & \multirow{6}{*}{$\begin{array}{l}\text { Vegetable } \\
\text { market, } \\
\text { Lahore, Cantt. }\end{array}$} & Bacillus farraginis & 08 & 25 \\
\hline & & & Kurthia gibsonii & 07 & 22 \\
\hline & & & Enterobacter agglomerans & 07 & 22 \\
\hline & & & Ralstonia solanacearum & 03 & 9.6 \\
\hline & & & Azospirillum lipoferum & 04 & 12 \\
\hline & & & Acinetobacter lwoffii & 02 & 6.4 \\
\hline \multirow[t]{5}{*}{03} & \multirow[t]{5}{*}{ Turnip } & \multirow{5}{*}{$\begin{array}{l}\text { Vegetable } \\
\text { market, } \\
\text { Lahore, Cantt. }\end{array}$} & Peptococcus sp. & 03 & 18 \\
\hline & & & Kurthia gibsonii & 04 & 25 \\
\hline & & & Acidovorax facilis & 02 & 12.5 \\
\hline & & & Ensifer adhaerens & 03 & 18 \\
\hline & & & Acinetobacter calcoaceticus & 04 & 25 \\
\hline \multirow[t]{4}{*}{04} & \multirow[t]{4}{*}{ Onion } & \multirow{4}{*}{$\begin{array}{l}\text { Vegetable } \\
\text { market, } \\
\text { Lahore, Cantt. }\end{array}$} & Bacillus farraginis & 04 & 25 \\
\hline & & & Enterobacter agglomerens & 03 & 18 \\
\hline & & & Curtobacterium albidum & 06 & 37 \\
\hline & & & Acinetobacter lwoffii & 03 & 18 \\
\hline \multirow[t]{5}{*}{05} & \multirow[t]{5}{*}{ Cucumber } & Vegetable & Acetobacter aceti & 04 & 24 \\
\hline & & Market, & Acidovorax temperans & 05 & 29 \\
\hline & & Lahore, Cantt. & Bordetella pertussis & 03 & 18 \\
\hline & & & Ensifer adhaerens & 02 & 12 \\
\hline & & & Enterobacter agglomerans & 03 & 18 \\
\hline \multirow[t]{4}{*}{06} & \multirow[t]{4}{*}{ Ginger } & \multirow{4}{*}{$\begin{array}{l}\text { Vegetable } \\
\text { market, } \\
\text { Lahore, Cantt. }\end{array}$} & Lactococcus lactis & 04 & 31 \\
\hline & & & Pantoea sp. & 03 & 23 \\
\hline & & & Spirillospora albida & 02 & 15 \\
\hline & & & Curtobacterium albidum & 04 & 31 \\
\hline \multirow[t]{7}{*}{07} & \multirow[t]{7}{*}{ Lemon } & Fruit market, & Bordetella pertussis & 04 & 22 \\
\hline & & \multirow{6}{*}{ Lahore, Cantt. } & Acinetobacter calcoaceticus & 03 & 17 \\
\hline & & & Ensifer adharrens & 04 & 22 \\
\hline & & & Aerococcus sp. & 01 & 05 \\
\hline & & & Kurthia gibsonii & 02 & 11 \\
\hline & & & Syntrophospora sp. & 01 & 05 \\
\hline & & & Burkholderia pseudomallei & 03 & 17 \\
\hline \multirow[t]{4}{*}{08} & \multirow[t]{4}{*}{ Spinach } & Vegetable & Micrococcus luteus & 03 & 25 \\
\hline & & market, & Enterobacter agglomerans & 02 & 17 \\
\hline & & Lahore, Cantt. & Proteus vulgaris & 04 & 33 \\
\hline & & & Bacillus farraginis & 03 & 25 \\
\hline 09 & Radish & Vegetable & Microbacterium lacticum & 02 & 20 \\
\hline & & market, & Lactococcus lactis & 03 & 30 \\
\hline & & Lahore, Cantt. & Corynebacterium minutissium & 03 & 30 \\
\hline & & & Bordetella pertussis & 02 & 20 \\
\hline 10 & Mint & Vegetable & Peptococcus sp. & 04 & 27 \\
\hline & & market, & Burkholderia pseudomallei & 03 & 20 \\
\hline & & Lahore, Cantt. & Ensifer adhaerens & 02 & 13 \\
\hline & & & Arthrobacter sp. & 03 & 20 \\
\hline & & & Bordetella pertussis & 03 & 20 \\
\hline
\end{tabular}


dominance of genera in the present study accorded with the results of (Hung and Annapurna 2004). Bacterial isolates from different agronomic plants and especially Enterobacter aggglomerans, Ensifer adhaerens and Bordetella pertussis those one with highest occurrence frequency showed that these are physiologically more versatile, not only in coping with the harshness of the climate but also have strong resistance to pollutants like heavy metals, herbicides, pesticides and antibiotics produced by other microorganisms and/or plants (Bajwa et al. 2009). Conversely, some species were isolated from a single locality, perhaps because they prefer a particular type of chemicals present in that locality or they lack competitive saprophytic ability to fight against antibiotics/ toxic substances produced by other flora and/or plants. Data also showed that percentage composition of bacterial flora was considerably influenced by the type of the plant (Table 2). The performance and stability of terrestrial ecosystems are determined by biodiversity and species composition. Distribution patterns of microorganisms provide important clues about the underlying mechanisms that structure ecological communities and are central to setting conservation priorities (Bajwa et al. 2009).

Bacterial strains isolated in the present investigation are a novel addition to the microdiversity of agricultural crops in Pakistan.

\section{References}

Ali A and Naseem F 2011. Isolation, cultivation, purification and identification of bacterial species from microfauna of soil. Ital. J. Public Health. 8(1): 34-39.

Ali A and Naseem F 2012. Frequency distribution of bacteria isolated from different industrial effluents Daffodil Int. Uni. J. Sci. Technol. 7(1): 28-33.

Bajwa R, Ibtasam K, Sobia M, Fozia N and Irum M 2009. Analysis of fungal diversity in rhizosphere of some plant species. Mycopath. 1(2): 5-10.

Benson H 1996. Microbiological applications, laboratory manual in general microbiology. W. M. C. Brown Publishers, Dubuque, U.S.A.

Holt J, Krieg N, Sneath P and Staley J 2000. Bergey’s Manual of Determinative Bacteriology. 9th ed. Williams \& Wilkins, Baltimore, USA: 175-189.

Hung P and Annapurna K 2004. Isolation and characterization of endophytic bacteria in Soybean (Glycine sp.). Omonrice 12: 92-101.

Ishaq F and Khan A 2011. Isolation, identification and comparative study of fungal and bacterial strains found in organic and inorganic soils of different agricultural fields. Recent Res. Sci. Technol. 3(11): 30-36.

Koneman W, Allen D, Janda M, Schreckenberger C and Winn J 1997. Atlas and textbook of diagonistic microbiology. 5th ed. Lippincott Williams \& Wilkins, 530 Walnut Street, Philadelphia, USA.

Zinniel K, Lambrecht A, Harris N, Feng Z, Kuczmarski D, Higley P, Ishimaru, Carol A, Arunakumari A, Barletta G and Vidaver M 2002. Isolation and characterization of endophytic colonizing bacteria from agronomic crops and prairie plants. Papers in Plant Pathol. Paper 40. http://digitalcommons.unl.edu/ plantpathpapers/40. 